\title{
Energy-Efficiency based Analysis of Routing Protocols in Mobile Ad-Hoc Networks (MANETs)
}

\author{
Parul Aggarwal \\ Research Student, Department of Computer \\ Engineering \\ Punjabi University, Patiala \\ Punjab- 147002 (India)
}

\author{
Himashu Aggarwal, Ph.D \\ Professor, Department of Computer Engineering \\ Punjabi University, Patiala \\ Punjab- 147002 (India)
}

\begin{abstract}
A mobile MANET (Mobile Ad-hoc Network) is an actively self organizing and self configuring network without the need of any centralized base station. This is a collection of various mobile nodes that is connected through a wireless medium forming rapidly changing topologies in the network. The MANETs are infrastructure less in nature and can be set up anytime, anywhere. In the MANETs, all the nodes are mobile and are battery operated. As because all the nodes in MANETs have limited battery resources and due to node mobility, multi hop routes are used over a changing network environment, so to limit the power consumption, to prolong the network lifetime and to improve the robustness of the system, energy efficient routing protocols are required. The design of efficient routing protocols is a fundamental problem in Mobile Ad-Hoc Network (MANET). Many routing protocols have been proposed in the literature, each one is based on different characteristics and properties in MANETs. Some of these respective protocols have been studied and their performances have been analyzed focusing on aspects like energy consumption, packet delivery fraction, network lifetime etc. In this paper, a performance comparison of the DSR (Dynamic Source Routing), AODV (Ad-Hoc on Demand Distance Vector) and DSDV (Destination Sequenced Distance Vector) routing protocols is evaluated on the basis of energy efficiency metrics by varying number of nodes, number of connections and pause time. Simulations are done using NS-2(version NS2.35). It has been verified through extensive simulations, which mostly represents a wide spectrum of network conditions that DSR delivers the better performance and needs smaller energy expenditure as that of algorithms AODV and DSDV but it is observed that DSDV prolongs the network lifetime better than the other two protocols and provides better load balancing capability.
\end{abstract}

\section{General Terms}

MANET, Energy efficiency analysis

\section{Keywords}

MANET, Routing Protocols, Energy Efficiency, Analysis, AODV, DSDV, DSR

\section{INTRODUCTION}

A mobile Ad-hoc network (MANET) is a collection of wireless mobile nodes that establishes the network dynamically in the absence of a fixed infrastructure. Ad-hoc is an imparted mode of communication which allows computers to directly interchange all the information with each other even without a router. In Latin, the word ad-hoc means "for this" [15] meaning "for this special purpose". MANET has one of the distinctive features that each node must have the ability to act as a router to find out the optimal path to forward a packet. As the nodes may be mobile, when they enter or leave the network, the topology of the network will change continuously. So in order to provide proper end-to-end communication all-over the network, peer hosts cooperate with each other to handle network functions needed for the communication, for ex. packet routing.

One of the most important research areas in MANETs is establishing and maintaining the Ad-hoc network by the use of routing protocols. Ad-hoc routing protocols can be divided into following categories as shown in Figure 1: proactive routing protocols and reactive routing protocols. Proactive (tabledriven) routing protocol [1] is an approach where each router can build its own routing table based on the information that each router or node can learn by exchanging information among the network's routers. Reactive (on-demand) routing protocol is an approach where the routing process needs to discover a route whenever a packet arrives from a source and needs to be delivered to a destination. These are sourceinitiated.

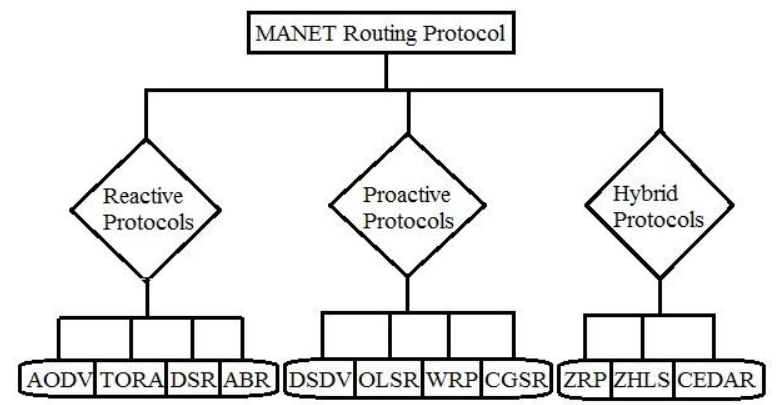

Figure 1: Classification of MANET routing protocols

Mobile Ad-hoc networks suffer many challenges during routing. Energy efficiency is the biggest challenge of an Adhoc network. In mobile Ad-hoc networks, all the mobile nodes rely on batteries for power and working, which are limited sources of energy for working, and, in cases of many environment conditions, it becomes very much a hectic task to replace or recharge them. So an Energy-efficient communication is critical for increasing the life of power limited wireless ad-hoc networks. A routing protocol should try to minimize control traffic so that the energy of the nodes and network can be managed effectively. Energy Management is defined as the process of managing the sources and consumers of energy in a node or in a network as a whole for enhancing the lifetime of the network [3].So if the network lifetime (time duration when the first node of the network dies) is to be increased as well the node lifetime then an efficient energy management protocol must be there. 
This paper considers analysing the performance comparison of AODV, DSDV and DSR routing protocols on the basis of energy efficiency metrics such as energy consumption, packet delivery fraction, network lifetime etc., so that a particular routing protocol can be selected as energy efficient and if required, further improvements could be made to the protocol to make it more energy efficient.

The rest of the paper is organized as follows. Section II describes the overview of selected routing protocols for simulations. Section III defines the problem statement. Section IV presents the performance metrics used. Section V concerned with the Simulations Methodology used and provides the results obtained from the simulations. Section VI concludes the paper.

\section{OVERVIEW OF ROUTING PROTOCOLS IN MANETS}

\subsection{Destination-sequenced distance vector (DSDV) [5]}

DSDV routing protocol is the one that comes under the category of proactive routing protocol. This is a distance vector routing protocol uses the bellmann-ford algorithm. DSDV also has the feature of hop-by-hop distance vector routing protocol in which every node maintains routing table listing the "next hope" and "number of hopes" information for each possible destination in the network. The periodical broadcasts of routing updates attempt to keep the routing table completely update at all the times [1]. In the routing table each entry has sequence number specified. So, whenever a new entry in a routing table has to be obtained, then the specific protocol prefers to select the entry with the largest sequence number assigned. If these entries with the same sequence number have been obtained, then the protocol selects the metric with the lowest value assigned.

Routing information is transmitted by broadcast. The routing updates have to be transmitted periodically or immediately when any significant topology change is available to it. The specific sequence numbers [5] are assigned by destination node, which means the destination gives a sort of default even sequence number specified, and the node emitter has to send out the next update with this number specific. The data packets are exchanged from the source to destination in the network by using routing table which are stored at the each station of the network in use. The routing information [8] is advertised by broadcasting or multicasting the packets which are transmitted periodically and incrementally as topological changes are detected - for some time specified, when the stations move within the network area. The data is also kept about the length of time between arrival of the first and the arrival of best route for each destination node. The respective entries in the routing table may change fairly dynamically over some certain time period.

\subsection{Dynamic source routing (DSR) [6]}

DSR protocol always comes under the category of an ondemand/reactive routing protocol. This is a simple and efficient routing protocol intended specifically for use in the multi-hop wireless Ad- hoc networks of mobile nodes present. DSR [6] also allows the network to be completely of a self-assembling and a self-arrangement nature, even without the need for any existing network infrastructure or administration thereby. This routing protocol uses explicit source routing which means that every time a data packet is sent along the network, it always accommodates the list of nodes it will use to be forwarded along. This protocol allows a route to be discovered dynamically across multiple network hops to any destination in the network. The source routing means that every packet in its header contains the complete sequential list of nodes through which the packet must pass by. The two main mechanisms are used in DSR protocols which are as specified: route discovery and route maintenance. These mechanisms will work together to allow the nodes to discover and maintain the routes to random destinations in the ad-hoc network in use. The DSR protocol also has many advantages over the routing protocols like AODV, LMR, and TORA and in small to moderately sized networks also, the DSR protocol performs better than all the above mentioned routing protocols also. There is no such periodic routing of messages in DSR routing protocol, thus helps in reducing the network bandwidth overhead, also the conservation battery power and in avoiding large routing updates throughout the ad-hoc network. In this process, the data packet contains the source route in packet header and routes are stored in memory used. There is not any routing loop in this protocol. If ever there is any data packet available to send over the network, and if it has no route, then the route discovery process is initiated successfully. The route discovery process of DSR is similar to the route discovery process of the AODV protocol.

Every node that receives the route request packet in network, broadcasts it, but except for the destination node or nodes that have route to destination node in their memory along. The route through network is built by RREQ packet sent, and then the RREP packet is being routed backward to the source node. The particular route that returns RREP packet is cached on the source node for any further use. There possibly can be multiple RREP packets on one RREQ packet generated [1]. During this sending process whenever broken link is detected in the communication, the RREQ packet has been sent backward to the source node in the network. When this RREQ packet has been received source node initiates another route discovery operation for communication. The routes that always contain the broken link should be removed from the route cache information.

\subsection{Ad-hoc on demand distance vector routing protocol (AODV) [10]}

The Ad-hoc on demand distance vector routing protocol, AODV is a very effective, simple and efficient routing protocol for Mobile Ad-hoc Networks. In AODV protocol, a no fixed topology is there for mobile ad-hoc networks. This is a loop free routing protocol of MANET. It surely enables the multihop routing between the participating mobile nodes wishing to establish and maintain an ad-hoc network [1]. This particular routing protocol is based on the distance vector algorithm mostly. This specific algorithm uses many different messages to discover and maintain links over the network. Whenever any particular node wants to try and find a route to another node it broadcasts a Route Request (RREQ) to all its neighbours in the network. The RREQ always passes through the network until it reaches the destination or the node with a fresh enough route to the destination node in the network. Then that particular route is made available by uncasing a RREP back to the source node in the network. This specific algorithm uses the hello messages, which is a special case of RREP message that are broadcasted periodically to the immediate neighbours. These kinds of hello messages [8] are local advertisements for the continued presence of the nodes in the network and the neighbours also using routes through the broadcasting node will continue to mark the routes as almost valid. If the hello messages anytime stop coming from a particular node over the network, then the neighbour can assume that the node has 
moved away and mark that link to the node as broken and notify the affected set of nodes by sending a link failure notification, which is also a special RREP case, to that set of nodes in the network.

\section{PROBLEM DEFINITION}

Mobile Ad-hoc networks suffer many challenges during routing. Energy efficiency is the biggest challenge of an Adhoc network. Energy efficiency can be measured as the amount of time over which the network can maintain a certain performance level, which is commonly known as the network lifetime. In mobile Ad-hoc networks, mobile nodes rely on batteries for power, which are limited sources of energy for them, moreover in many sort of environments, it becomes quite a hectic task to replace or recharge them. Energy from these batteries is consumed in many processes and from many sources during routing process. Communication is one of the main sources of energy consumption. Every message that is sent and every computation performed by the nodes drains the battery. Some amount of energy is lost even when a node is in idle mode. Therefore, Energy-efficient communication is critical for increasing the life of power limited wireless ad-hoc networks. A routing protocol should try to minimize the control traffic occurring, for ex. the periodic update messages to improve the lifetime of the nodes and network and so that the energy of the nodes and network can be managed effectively. Energy Management is defined as the process of managing the sources and consumers of energy in a node or in a network as a whole for enhancing the lifetime of the network [3]. So if the network lifetime (time duration when the first node of the network dies) is to be increased as well the node lifetime then an efficient energy management protocol must be there.

So in this paper, the performance of certain routing protocols on the basis of their energy efficiency has been analyzed, so that a particular routing protocol can be selected as energy efficient and if required, further improvements could be made to the protocol to make it more energy efficient.

\section{PERFORMANCE METRICS}

The performance metrics used for performance analysis of the routing protocols are as follows:

\subsection{Energy Consumed per Packet Delivered (ECPD)}

It is defined as the ratio of total network energy consumption to the number of data packets successfully received by the source. The network energy consumption [9] includes all the energy consumptions except MAC layer controls. Energy consumption is formulated as the difference between the initial energy of the node and the remaining energy of the node at the end of simulation. A less value of this metric means that most of the packets being received with less energy and is a sign of achievement for an energy efficient protocol.

\subsection{Network Lifetime (NL)}

It is one of the important metrics used to evaluate the energy efficiency of the routing protocols with respect to network partition [9]. In wireless Ad-Hoc networks, especially in which the nodes are distributed densely, the death of the first node seldom leads to the total failure of the network. When the number of dead nodes keeps increasing, the network gets partitioned. But even when the network gets partitioned, endto-end transmissions may still be possible, if there exist at least one pair of adjacent nodes working which keep the network alive, since they could transmit to each other thus keeping the network to be working. So, lifetime of the network may be defined as the time until the first node in the network dies (drains-out) or the time when all the nodes in the network die. A node with less than $10 \%$ of its full battery capacity is considered as a dead node [9].

\subsection{Packet Delivery Fraction (PDF)}

It is defined as the ratio of total number of packets successfully received by the destination nodes to the number of packets sent by the source nodes. PDF [9] is very important metric as it describes the loss rate i.e. it gives the maximum throughput that the network can support. There may be many reasons due to which packets may not be delivered to the destination such as packet collisions may occur in the layer, network partitions, routing loop, interface queue drop etc. A high value of PDF indicates [9] that most of the packets sent are being delivered to the higher layers and is a positive sign of the protocol performance.

\subsection{Variance in Residual Battery Energy (VRBE) of nodes}

It is a simple indication of energy balance [12] and can be used to extend network lifetime. The idea behind this metric is that all mobiles in a network operate at the same priority level. In this way, all mobiles are equal and no one mobile is penalized or privileged [2] over any other node in the network. This metric also makes sure that all the mobiles in the network remain powered-on together for as long as possible. The value calculated indicates [9] whether the routing scheme has overused any number of nodes or not. This is a very important performance metric because it is a measure of protocol fairness. Therefore, the sought value for this metric is one that is as close as possible to 0(zero). The smaller value of this metric indicates that all nodes in the network are equally important and no one node must be penalized more than any of the other nodes.

\section{SIMULATION METHODOLOGY AND RESULTS}

In this research work, a Discrete Event Simulator is being used called Network Simulator-2(NS-2.35) [13] [14] for simulating the energy efficiency of AODV, DSDV and DSR Protocols. Discrete Event Simulation is a software-based method to employ the models of real environment to draw a conclusion from the output.

\subsection{Simulation Framework and Scenarios}

The basic methodology in this work consist of first selecting the most representative parameters for a MANET, then defining and simulating a basic Scenario and finally by varying the selected parameters, simulate and evaluate more Scenarios. The selected parameters are: 1) Number of Mobile Nodes, 2) The Pause Time and 3) The Number of Data Connections.

In this work, two Scenarios have been considered for evaluation of the protocols. In Scenario-1, 25 Mobile nodes are considered for simulations and in Scenario-2, 50 Mobile nodes are considered. Further evaluations are performed by varying the parameters by considering two cases. In the first case, the number of data connection parameter is varied keeping the pause time constant to 4 seconds, and in the second case, the pause time parameter is varied keeping the number of connections constant to 20 connections.

The simulation parameters for the research work are shown in Table 1. 
Table 1: Simulation parameters used for Simulations

\begin{tabular}{|l|l|}
\hline Parameters & Values \\
\hline No. of Nodes & 25,50 \\
\hline No. of Connections & $5,10,15,20,25,30$ \\
\hline Simulation time & 500 seconds \\
\hline Environment size & $670 \times 670 \mathrm{~m}^{2}$ \\
\hline Node Speed & $10 \mathrm{~m} / \mathrm{s}$ \\
\hline Routing Protocols & AODV, DSDV, DSR \\
\hline Mobility model & Random Waypoint \\
\hline Pause time & $4,100,200,300,400,500 \mathrm{sec}$ \\
\hline Propagation Model & Two Ray Ground \\
\hline Traffic model & CBR \\
\hline Traffic size & 4 packet/sec \\
\hline Packet size & 512 bytes \\
\hline
\end{tabular}

\subsection{Mobility Model}

In this research work, the mobility model considered is the Random Waypoint Mobility Model. It is known to be one of the most used models by researchers. The Random Waypoint Mobility Model [11] includes pause times between changes in direction and/or speed. A node begins by staying in one location for a certain period of time (i.e., a pause time). Once this time expires, the mobile node chooses a random destination as well as a speed that is uniformly distributed between [MINSPEED, MAXSPEED]. It then travels towards the newly chosen destination at the selected speed. Upon arrival, the node takes another break before starting the process again.

\subsection{Energy Model}

Energy Model [8] is a node attribute that represents level of energy in a mobile node [4]. The basic energy model is determined by Class EnergyModel in NS-2 with following attributes:

-txPower : Transmitting power (watts)

-rxPower: Receiving power (watts)

-initialEnergy: Starting Energy (joules)

-idlePower: When node is sitting idle and listening (watts)

-sleepPower: when radio is turned off and node is not capable of detecting signals (watts)

The following equations [7] represent the energy used (in Joules) when a packet is transmitted (Eqn.1) or when received (Eqn. 2):

$$
\begin{array}{ll}
\text { Energy }_{\mathrm{tx}}=(330 * 5 * \text { PacketSize }) / 2 * 10^{6} & \text { Eqn.1 } \\
\text { Energy }_{\mathrm{rx}}=(230 * 5 * \text { PacketSize }) / 2 * 10^{6} & \text { Eqn. 2 }
\end{array}
$$

The energy model parameters used in the simulation are shown in Table 2.

Table 2: Energy model Parameters

\begin{tabular}{|l|l|}
\hline Parameter & Value \\
\hline Energy Model & Energy Model \\
\hline Initial Energy & 1 Joule \\
\hline Receiving power & $0.1 \mathrm{~W}$ \\
\hline Transmission power & $0.2 \mathrm{~W}$ \\
\hline Idle power & $0.01 \mathrm{~W}$ \\
\hline Sleep power & $0.01 \mathrm{~W}$ \\
\hline Transition Power & $0.1 \mathrm{~W}$ \\
\hline Transition time & 0.01 \\
\hline
\end{tabular}

\subsection{Results and discussions}

\subsubsection{Effect of varying the number of connections}

5.4.1.1 Scenario-1 (25 nodes)

The Figures for Scenario-1 where the parameter number of connections is varied are shown in Figures 2, 3, 4 and 5. Figure 2 shows the packet delivery fraction of AODV, DSDV and DSR with 25 mobile nodes with respect to the number of connections.

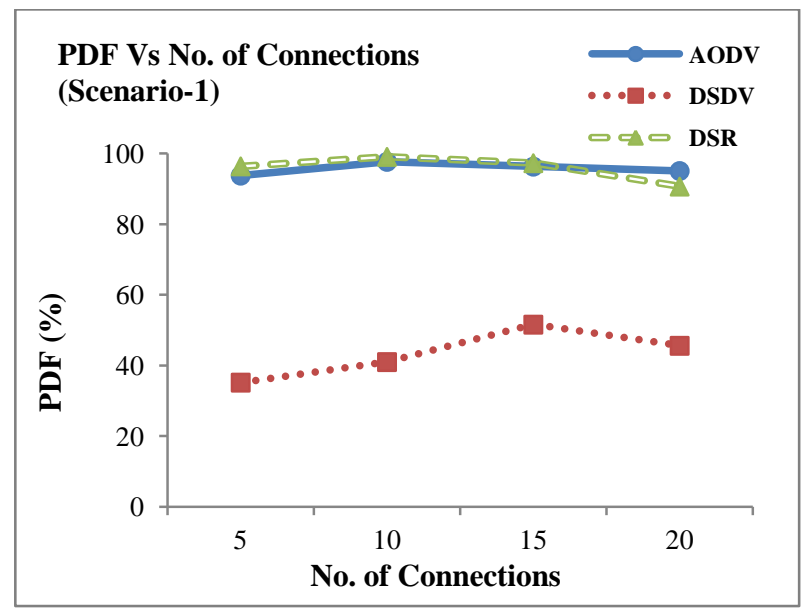

Figure 2: Scenario-1 (25 nodes) PDF Vs No. of Connections

PDF of DSDV is almost of the same value for all number of connections, due to the fact that DSDV keeps a constant routing overhead in any number of traffics. The DSDV routing overhead depends only on the periodic update interval and the size of the network. DSR and AODV adapt over 90\% of PDF, for less number of connections. Their performance does not remain same for large number of connections. The PDF of the DSR is better than AODV mostly for all number of connections.

Figure 3 shows the network life time of the three MANETs routing protocols. The network lifetime is the cumulative results of ECPD and VRBE metrics. DSDV routing protocol makes the network energetic for a longer time than the others two, due to the fact that DSDV energy consumption per packet is less than DSR and AODV. Moreover, the balance of energy consumption in DSDV is better than AODV and DSR as shown in the Figure 5. In DSDV, all nodes exchange the routing update whether they are active or inactive periodically. But AODV and DSR use only the active nodes for route establishment. This causes particular nodes to get repeatedly engaged in route discovery and data packet transmission. This restricts load balancing. DSR has longer network life than AODV. But AODV gives better load balancing than DSR as shown in Figure 5. The network life time is shown by less energy consumption and appropriate load balancing. Here, the ECPD of DSR dominates the load balance of AODV for this Scenario. 


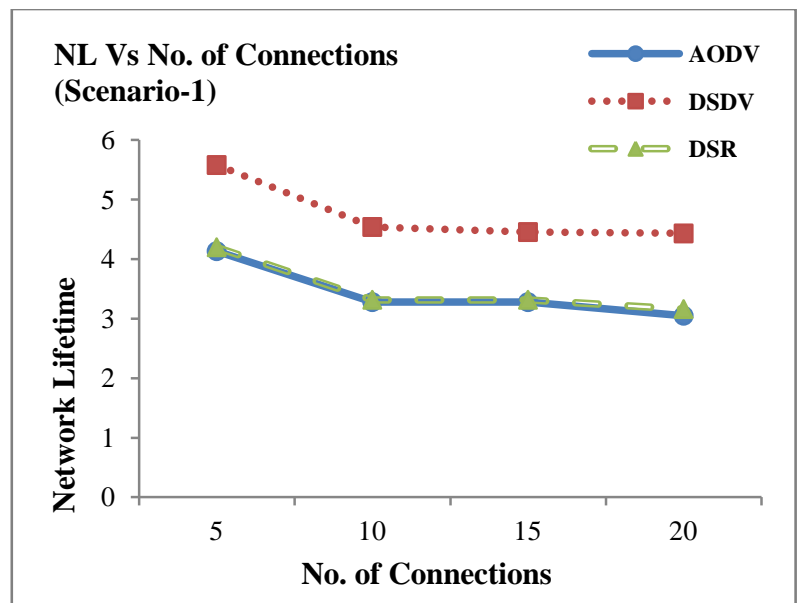

Figure 3: Scenario-1 (25 nodes) Network Lifetime Vs No. of Connections

Figure 4 shows the ECPD of AODV, DSDV and DSR. DSR uses less ECPD than AODV and DSDV. In dynamic network, DSDV doesn't have up to date route entry to send the data packets. The node sends all the packets on staled out routes. The packet is transmitted successfully after few attempts. These attempts lead to much energy consumption. Moreover, DSDV requires the periodic transmission of reach ability information; this also leads to higher energy consumption of DSDV. DSDV consumes more energy than AODV. The energy consumption of these routing protocols increases as the numbers of connections increase. Mainly, ECPD of DSR increases dramatically as the numbers of connections increases, due to the fact that DSR caches large number of routes for large number of connections. But these routes are not valid for much time due to high mobility.

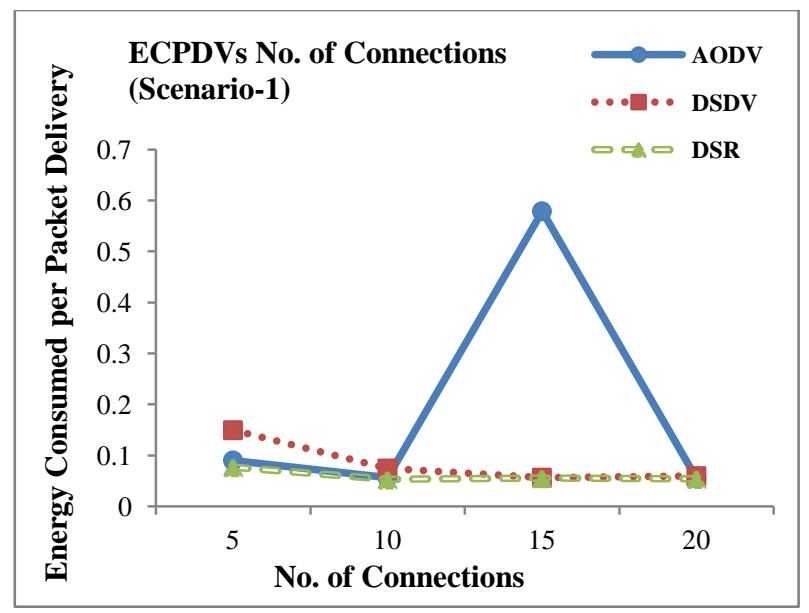

Figure 4: Scenario-1 (25 nodes) Energy Consumed per Packet Delivery Vs No. of Connections

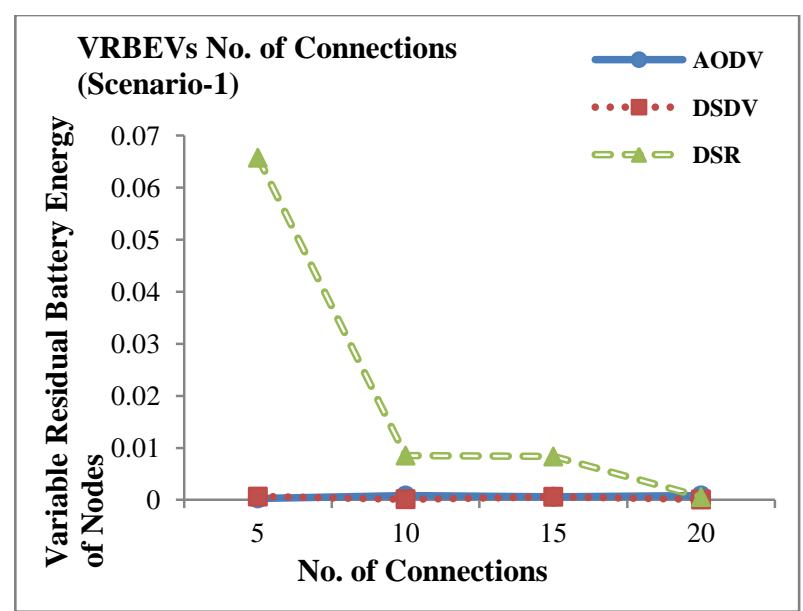

Figure 5: Scenario-1 (25 nodes) Variable Residual Battery Energy of nodes Vs No. of Connections

5.4.1.2 Scenario-2 (50 nodes)

The Figures for Scenario-2 in which number of connections is varied are shown in Figures 6, 7, 8, and 9.

Figure 6 shows the metric PDF for Scenario-2, in which DSR and AODV outperform DSDV. PDF of DSR is better than that of AODV because DSR has access to more amount of routing information than AODV in a single cycle of route discovery. Due to the source routing in DSR, along-with the intended destination, the source can also learn routes to each intermediate node on the route, using a single request-reply cycle. DSR can also get access to a significant amount of routing information because of promiscuous listening on data packet transmissions. AODV can gather small amount of routing information in the absence of source routing and promiscuous listening. So AODV uses too many routing packets for accessing number of routing information as in DSR.

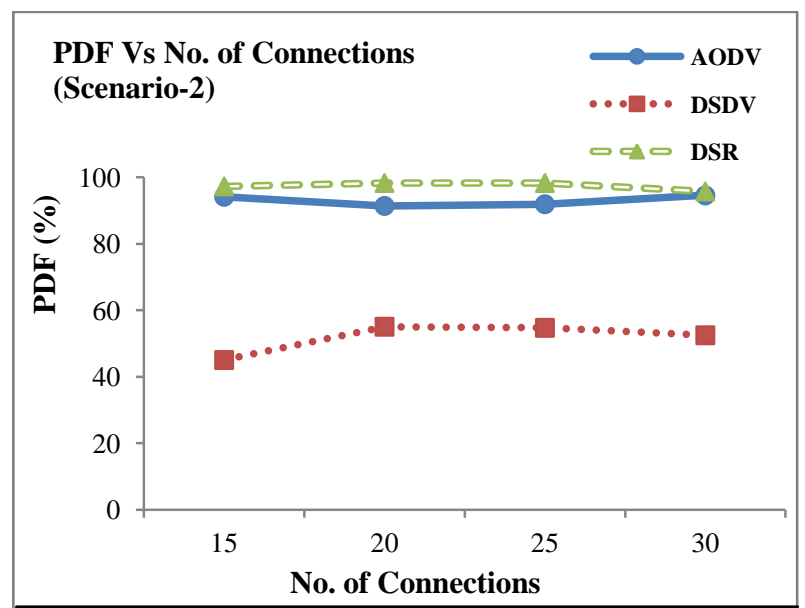

Figure 6: Scenario-2 (50 nodes) PDF Vs No. of Connections

The network lifetime of these three MANETs routing protocols is demonstrated in Figure 7. DSDV gives longer network life time than DSR and AODV as shown in both the cases in Scenario-1. DSR gives longer network lifetime better than AODV. The link breakage because of high mobility is more serious in AODV than DSR because AODV is aggressive to maintaining broken links. Therefore, the network lifetime of AODV is shorter than DSR.

As shown in the Figure 8, DSR uses less ECPD than AODV and DSDV. DSDV consumes more energy than AODV. 


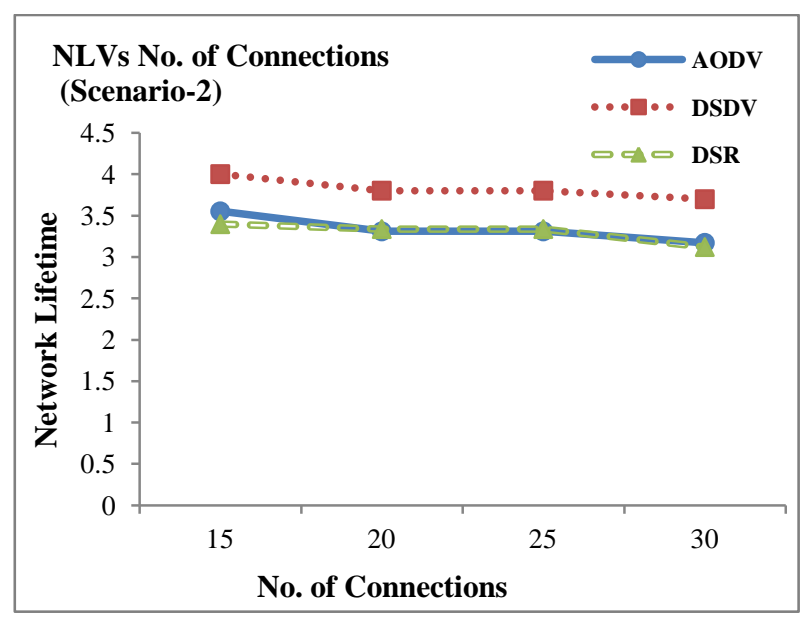

Figure 7: Scenario-2 (50 nodes) Network Lifetime Vs No. of Connections

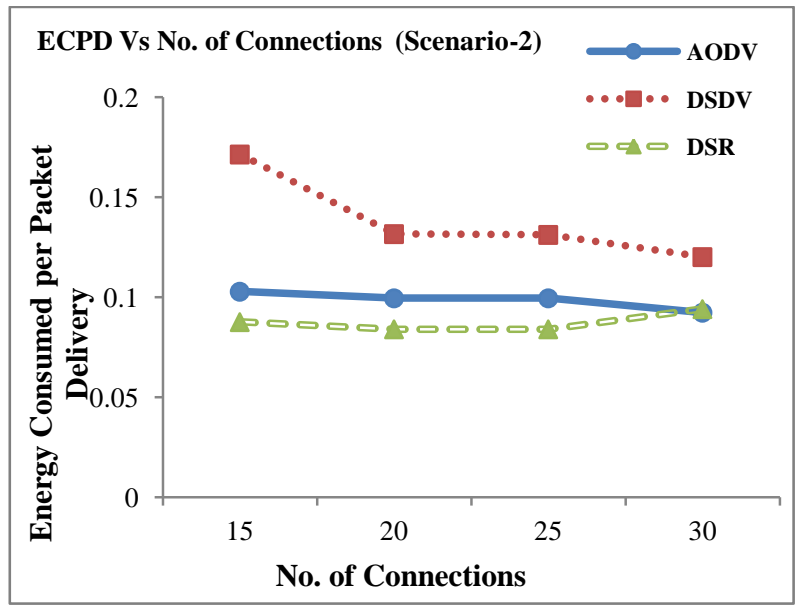

Figure 8: Scenario-2 (50 nodes) Energy Consumed per Packet Delivery Vs No. of Connections

Figure 9 shows the VRBE of these protocols for Scenario-2. AODV performs better than DSDV and DSR in case of this metric, as opposed to the previous Scenario, where DSDV showed better load balancing in both the cases. DSDV performs better load balancing than AODV in this case.

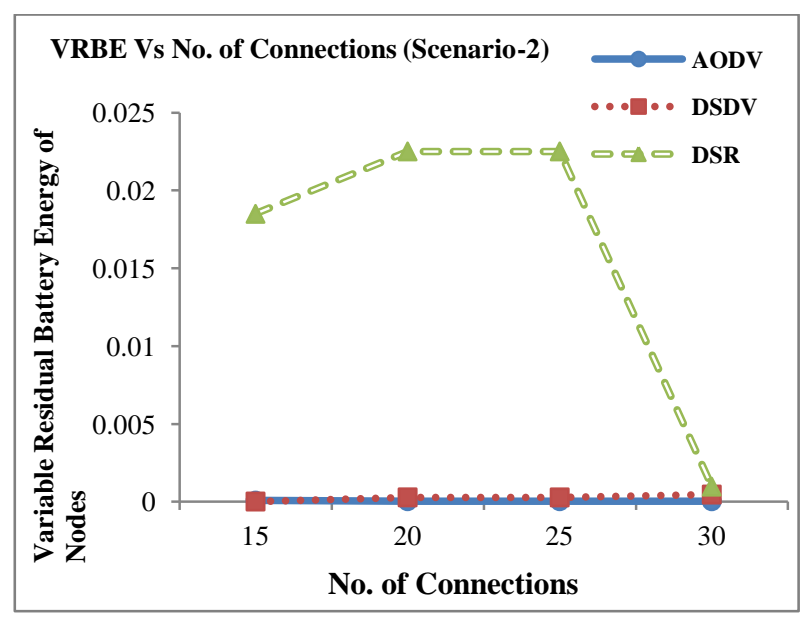

Figure 9: Scenario-2 (50 nodes) Variable Residual Battery Energy of nodes Vs No. of Connections

\subsubsection{Effect of varying the pause time}

\subsubsection{Scenario-1(25 nodes)}

The Figures for Scenario-1 where the parameter pause time is varied are shown in Figures 10, 11, 12 and 13.

Figure 10 shows the packet delivery fraction of the protocols for 25 nodes with respect to pause time. A smaller value of pause time means that the nodes will stop for smaller times and as a result, the routes will not be stable ever.

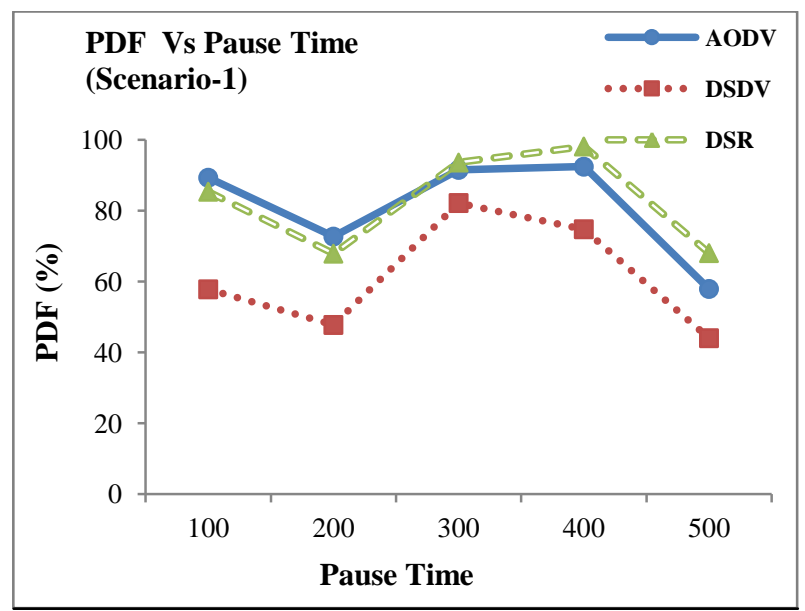

Figure 10: Scenario-1 (25 nodes) PDF Vs Pause Time

In this work, the maximum speed was kept at $10 \mathrm{~m} / \mathrm{s}$ and packet rate 4 packets/s. The results for 100, 200, 300, 400 and 500 seconds of pause time is shown. Here it can be seen that for a less value of pause time i.e. in relatively dynamic network, AODV gives more PDF but as the pause time value increases, i.e. in relatively a static network, DSR shows better PDF.

Figure 11 shows the network lifetime for 25 nodes. In this case also, DSDV gives a better network lifetime than the other two protocols as can be seen in the previous case.

Figure 12 shows the energy consumption per packet delivery of the protocols. In this case DSDV shows lesser consumption of energy than the other two protocols whereas the energy consumption of AODV and DSR are mostly equal.

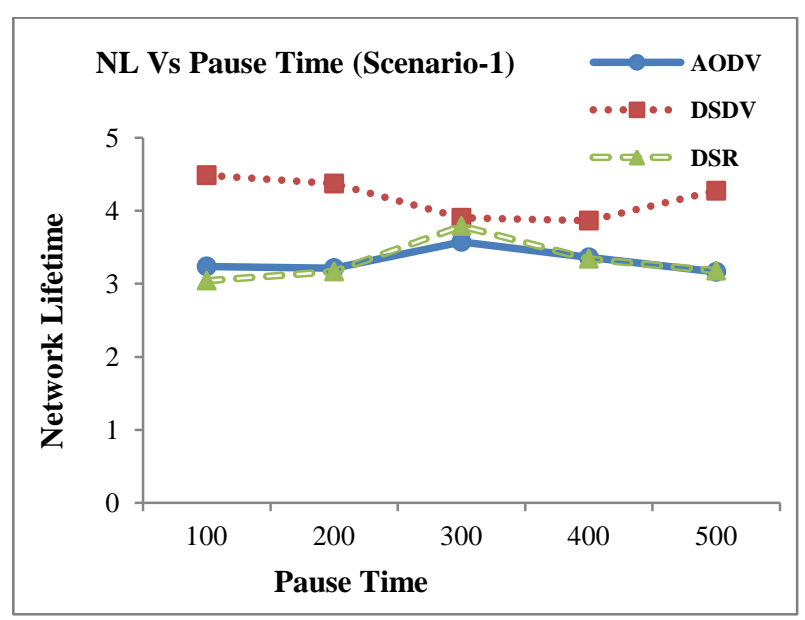

Figure 11: Scenario-1 (25 nodes) Network Lifetime Vs Pause Time 


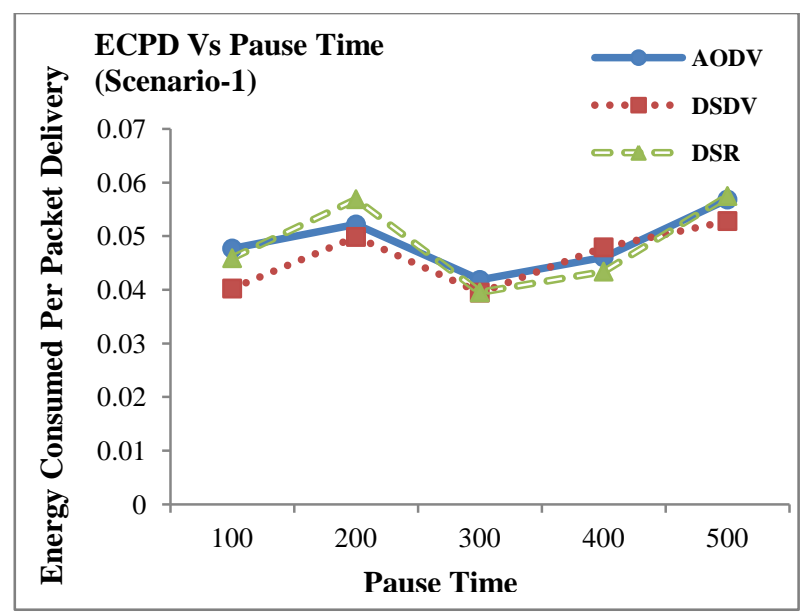

Figure 12: Scenario-1 (25 nodes) Energy Consumed per Packet Delivery Vs Pause time

Figure 13 shows the VRBE values of the protocols for 25 nodes. In this case also, DSDV gives better residual energy values than the other two protocols, as was seen in the previous case.

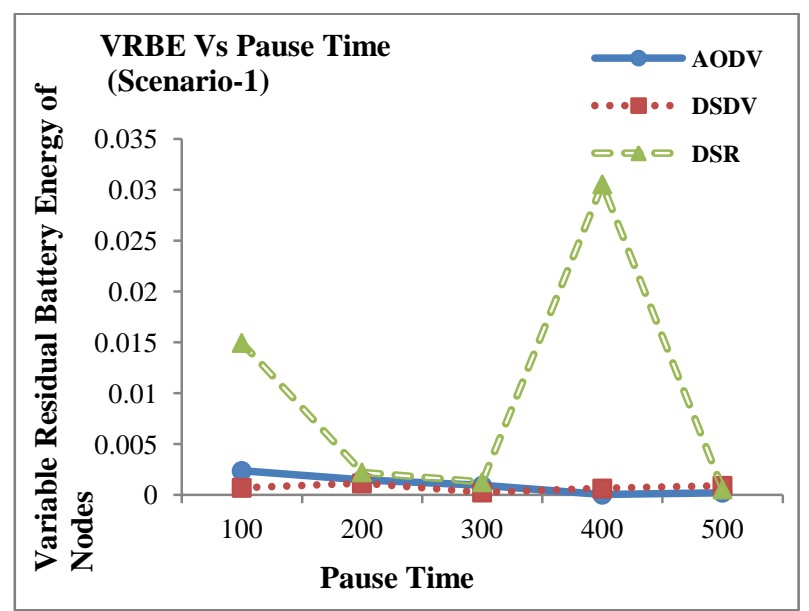

Figure 13: Scenario-1 (25 nodes) Variable Residual Battery Energy of nodes Vs Pause time

\subsubsection{Scenario-2(50 nodes)}

The Figures for Scenario-2 in which pause time is varied, are shown in Figures 14, 15, 16 and 17.

Figure 14 shows the values of PDF for the protocols in Scenario-2. It can be seen that DSR gives better value of PDF as in Scenario-1 and in the previous case. On the other hand, AODV gives better PDF than DSDV in this case.

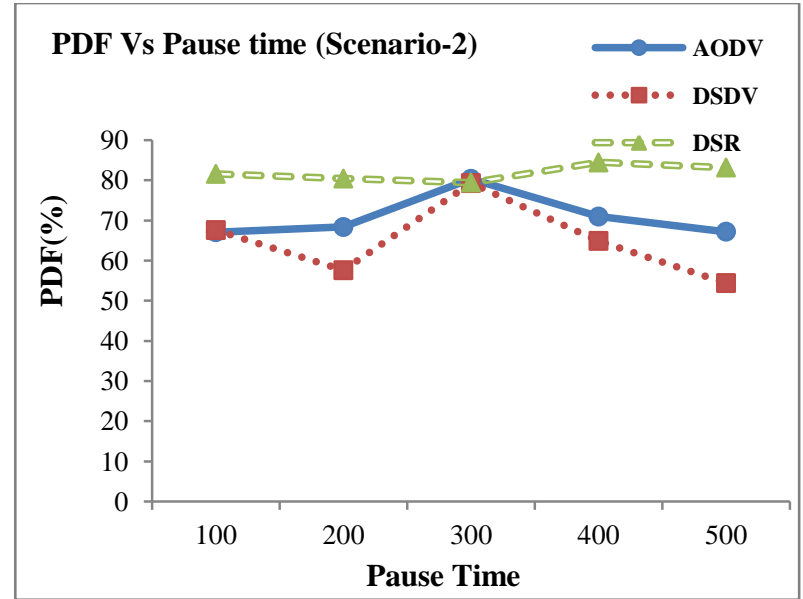

Figure 14: Scenario-2 (50 nodes) PDF Vs Pause time

Figure 15 shows the network lifetime of the protocols and as seen in previous cases, DSDV gives longer network lifetime than the other two protocols.

Figure 16 shows the energy consumed by the protocols. It can be seen that DSR shows lesser energy consumption for more static network, whereas DSDV gives better energy consumption for more dynamic networks.

Figure 17 shows the value of VRBE for Scenario-2. As shown, AODV performs better in this case, as seen in the previous case but opposed to the Scenario-1, where DSDV showed better value for VRBE. In this case also, DSDV shows better VRBE than DSR for dynamic networks and for static networks, DSR shows better load balancing than DSDV in this case.

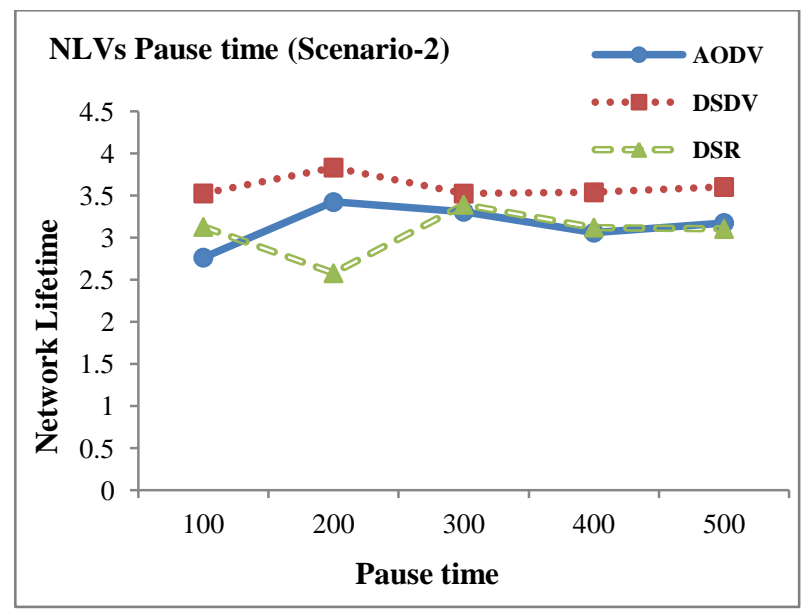

Figure 15: Scenario-2 (50 nodes) Network lifetime Vs Pause time 


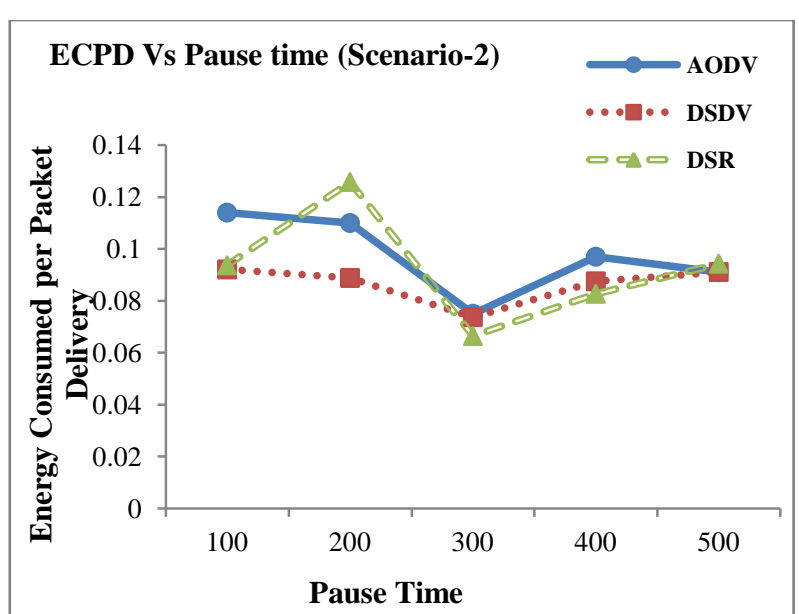

Figure 16: Scenario-2 (50 nodes) Energy Consumed per Packet Delivery Vs Pause time

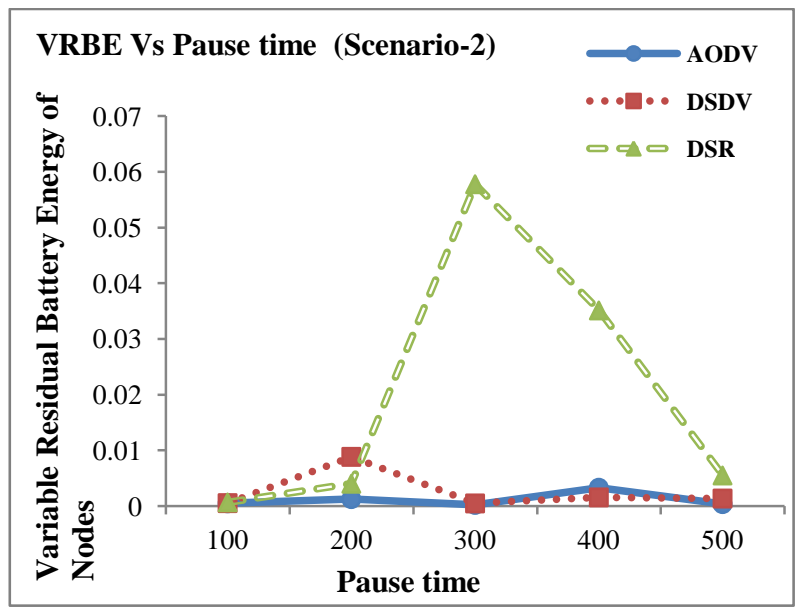

Figure 17: Scenario-2 (50 nodes) Variable Residual Battery Energy of nodes Vs Pause time

\section{CONCLUSIONS}

In the years to come, the topic of mobile computing will be flourishing, and it appears to be inevitable that there is an eventual seamless integration of MANET with other wireless networks, and the fixed Internet infrastructure. The importance and the crucial opportunity of ad-hoc networks is being increasingly recognized by both the research and industry community, as evidenced by the flood of research activities performed in this field so far, as well as all the exponential growth in the Wireless LANs and Bluetooth sectors done almost.

In the work in this thesis, the results of measuring and evaluating the energy consumption behaviour of three routing protocols; respectively Ad-hoc On Demand Distance Vector (AODV), Direct Source Routing (DSR) and DestinationSequenced Distance- Vector Routing (DSDV) are presented. The parameters for a MANET were selected which were the most representative, then two Scenarios and two cases were designed and simulated, and finally by varying the selected parameters, more scenarios were designed and results were generated.

The performance of AODV, DSR and DSDV routing protocols is analyzed with simulation using NS-2.35 simulator scenario available, by varying the number of nodes, the pause time and the number of connections on the basis of four performance and energy efficiency parameters viz. Packet Delivery Fraction (PDF), Network Lifetime (NL), Energy Consumption per
Packet Delivered (ECPD) and Variable Residual Battery Energy of nodes (VRBE).

The existing MANETs familiar routing protocols have not been designed to provide energy efficient route. These have been designed to provide their best efforts of less delay. Even if the design objectives of these protocols may be not to offer energy efficient route, still they have shown significant differences in energy conservation. A single routing protocol in MANETs may not be qualified to all the metrics of energy efficient routing. The processes of route discovery and maintenance of each of the routing protocol outperform for different energy efficient metrics.

Results show that DSR performs better in case of Energy Consumption per Packet Delivered and Packet Delivery Fraction, because of its less routing overhead. DSR consumes minimum energy than the other two protocols, due to the fact that DSR is advantageous from the cached route reply. The feature of cached route reply is used to reduce the broadcast storms. A number of multiple routes can be provided with a single cycle of route discovery in case of DSR. This ultimately reduces the energy consumption to get the route discoveries. DSDV prolongs the network lifetime better than DSR and AODV, due to the fact that DSDV is advantageous from its load balancing of routing overhead. In case of VRBE, DSDV performs better for less number of nodes, due to the fact that all nodes exchange the update whether they are active or inactive periodically, and as the number of nodes increase, AODV also gives good VRBE, because of its on-demand characteristics to determine freshness of the routes and as the number of nodes increases, then the active nodes for engagement in route discovery and data packet transmission also increases, thus giving more a good amount of load balancing. The network life time of AODV, DSR and DSDV comes down when the connections from source to destination increase. This is due to the fact that the collision rate and retransmission attempts increase for high traffics.

It was observed that increasing the number of nodes also increases energy consumption due to routing control packets. The energy consumption can be reduced by reducing the number of routing control packets to increase the lifetime of the network. In general case, the DSR protocol outperforms in many scenarios and metrics. Therefore it can be concluded that DSR is more energy efficient than DSDV and AODV with better performance. However, for a network with lesser number of nodes, a combination protocol of DSR and DSDV can be utilized to get the maximum network lifetime and reduce the energy consumption. Whereas, for a network with greater number of nodes, a combination protocol of DSR and AODV can be used for maximum performance and minimum energy consumption.

In the future work, the performance of other categories of routing protocols such as ZRP, OLSR, TORA etc., will be evaluated and measured under these Scenarios and an algorithm will be developed for reducing the energy consumption and prolonging the network lifetime.

\section{REFERENCES}

[1] Gupta, R. (2013). Ad-hoc routing protocol "review paper". Journal of Global Research in Computer Science, 4(5), 26-29

[2] Jones, C. E., Sivalingam, K. M., Agrawal, P., \& Chen, J. C. (2001). A survey of energy efficient network protocols for wireless networks. Wireless networks, 7(4), 343-358 
[3] Taneja, S., \& Kush, A. (2012). Energy efficient, secure and stable routing protocol for MANET. Global journal of computer science and technology, 12(10-E), 25-38

[4] Jung, S., Hundewale, N., \& Zelikovsky, A. (2005). Energy efficiency of load balancing in MANET routing protocols. In Software Engineering, Artificial Intelligence, Networking and Parallel/Distributed Computing, 2005 and First ACIS International Workshop on Self-Assembling Wireless Networks. SNPD/SAWN 2005. Sixth International Conference, IEEE, 476-483

[5] Perkins, C. E., \& Bhagwat, P. (1994). Highly dynamic destination-sequenced distance-vector routing (DSDV) for mobile computers. In ACM SIGCOMM Computer Communication Review, ACM, 24(4), 234-244

[6] Johnson, D. B., \& Maltz, D. A. (1996). Dynamic source routing in ad hoc wireless networks. In Mobile computing, Springer US, 153-181

[7] Cano, J. C., \& Manzoni, P. (2000). A performance comparison of energy consumption for mobile ad hoc network routing protocols. In Modeling, Analysis and Simulation of Computer and Telecommunication Systems, 2000. Proceedings. 8th International Symposium, IEEE, 57-64

[8] Feeney, L. M. (2001). An energy consumption model for performance analysis of routing protocols for mobile ad hoc networks. Mobile Networks and Applications, 6(3), 239-249
[9] Bohari, H. Y., \& Khanna, S. O. (2013). Energy Efficient Power Aware Routing Algorithm (EEPARA) For Mobile Ad Hoc Network (MANET). International Journal of Engineering, 2(7), 1562-1572

[10] NWG, "Ad-hoc On Demand Distance Vector Routing (AODV)", http://tools.ietf.org/pdf/rfc3561.pdf

[11] Davies, V. A. (2000). Evaluating mobility models within an ad hoc network (Master's thesis, advisor: Tracy Camp, Dept. of Mathematical and Computer Sciences. Colorado School of Mines)

[12] Mathews, D., Kannan, S., \& Karthik, S. (2012). A Performance Analysis of Energy Aware and Link Stability based Routing Protocols for MANET. International Journal of Advanced Research in Computer Engineering \& Technology (IJARCET), 1(8), 171-175

[13] NS2, http://www.isi.edu/nsnam/ns/doc/ns_doc.pdf

[14] NS2 Tutorial, http://www.isi.edu/nsnam/ns/tutorial

[15] Vadhwani, D. N., Kulhare, D., \& Singh, M. (2013). BEHAVIOUR ANALYSIS OF DSR MANETP ROTOCOL WITH HTTP TRAFFIC USING OPNET SIMULATOR.International Journal of Innovative Research in Computer and Communication Engineering, 1(3), 680-687 